\title{
BMJ Open Experiences participating in a community-based exercise programme from the perspective of people living with HIV: a qualitative study
}

\author{
Chantal A Montgomery, ${ }^{1}$ Katherine J Henning, ${ }^{1}$ Sarah R Kantarzhi, ${ }^{1}$ \\ Tamar B Kideckel, ${ }^{1}$ Cheryl F M Yang, ${ }^{1}$ Kelly K O'Brien ${ }^{1,2,3}$
}

To cite: Montgomery CA, Henning KJ, Kantarzhi SR, et al. Experiences participating in a communitybased exercise programme from the perspective of people living with HIV: a qualitative study. BMJ Open 2017;7:e015861.

doi:10.1136/bmjopen-2017015861

- Prepublication history and additional material is available. To view please visit the journal (http://dx.doi.org/ 10.1136/bmjopen-2017015861).

CAM, KJH, SRK, TBK and CFMY contributed equally.

Received 5 January 2017 Revised 23 February 2017 Accepted 1 March 2017

CrossMark

For numbered affiliations see end of article.

Correspondence to Dr Kelly K O'Brien; kelly.obrien@utoronto.ca

\section{ABSTRACT}

Objectives: Our aim was to explore the experiences of engaging in a community-based exercise programme (CBEP) from the perspective of people living with HIV (PLWH).

Design: We conducted a descriptive qualitative study using semistructured interviews.

Setting: We recruited adults living with HIV who participated in a 16-week CBEP in Toronto, Canada.

Participants: 11 participants, the majority men $(64 \%)$, with a median age of 52 years, and living with a median of 5 concurrent health conditions in addition to HIV participated in the study.

Outcome measures: We asked participants about their overall experiences: strengths, limitations and perceived benefits of the CBEP; factors influencing participation and current level of exercise after completion of the CBEP. We administered a selfreported demographic questionnaire followed by the Rapid Assessment of Physical Activity (RAPA) questionnaire. We analysed interview data using thematic analysis.

Results: We developed a framework that describes the experiences before, during and after the CBEP; and the perceived impact of the CBEP on health, which influenced the intent to, engagement in and sustainability of exercise among PLWH. Participants described the positive impact of the CBEP on their physical, mental and social health. Interviews were completed at a median of 6 months after the CBEP, when 9 participants reported ongoing engagement in exercise, but to a lesser extent than during the CBEP. Intrinsic and extrinsic factors facilitated or hindered engagement in exercise throughout all phases of the CBEP. The episodic nature of HIV and multimorbidity influenced engagement in exercise and posed challenges to re-engagement after periods of inactivity.

Conclusions: CBEPs provide an opportunity to enhance physical activity, perceived health outcomes and knowledge about exercise for PLWH.

Community-based exercise is a strategy that may be used by health providers to promote engagement in sustained physical activity for PLWH.

\section{Strengths and limitations of this study}

- To the best of our knowledge, this was the first qualitative study to explore the experiences of participating in a community-based exercise programme (CBEP) from the perspectives of adults living with HIV.

- Using a qualitative approach involving semistructured interviews enabled participants to describe their in-depth experiences following participation in a CBEP, their perceptions about the facilitators and barriers to engaging in a CBEP; perceived benefits on health and disability outcomes and their long-term engagement in exercise over time.

- Our team-based approach including concurrent data collection and analysis, which guided ongoing revision of the interview guide, and independent coding of each transcript by at least two authors enhanced the procedural and analytical rigour of the study.

- The majority of participants completed the CBEP and thus may have represented a 'healthy' community-dwelling sample of PLWH. Participants were primarily men living in an urban centre who completed the CBEP and agreed to participate in an interview. Hence, the transferability of these findings to women, people living with HIV in rural settings and to those who withdrew or were lost to follow-up is less clear.

\section{INTRODUCTION}

With increased access to combination antiretroviral therapy, people living with HIV (PLWH) are living longer with the complex and multidimensional health-related challenges attributed to HIV, ageing and multimorbidity, a concept known as disability. ${ }^{1-3}$ Disability can be experienced as multidimensional and episodic in nature. Disability can include health challenges such as physical (eg, joint pain, weakness, headaches), 
cognitive (eg, difficulty remembering; focusing attention), and mental and emotional (eg, feeling anxious) symptoms and impairments; difficulties carrying out day-to-day activities (eg, mobility, self-care); challenges to social inclusion (eg, engaging in personal relationships, work and leisure activities) and uncertainty and worrying about future health (eg, worrying about when an episode of illness might occur). ${ }^{4}{ }^{5}$ Hence, there is a need to address the prevalence and impact of disability for PLWH. ${ }^{6}$

Exercise is a self-management strategy that can address disability and improve strength, body composition and psychological outcomes for PLWH. ${ }^{7-10}$ Despite the benefits, few PLWH engage regularly in exercise compared to the general population. ${ }^{11}$ Stigma, lower socioeconomic status, lack of awareness and education regarding the benefits of exercise and transportation issues can pose barriers for older adults and individuals with stroke and may similarly pose barriers to exercise participation for PLWH. ${ }^{12-14}$ It is critical to consider novel ways to promote engagement in physical activity for PLWH.

Community-Based Exercise Programmes (CBEPs) offer a safe and effective strategy to reduce disability for PLWH. A CBEP includes a structured set of exercises designed for individuals with specific exercise needs; they commonly involve a group of persons with similar conditions exercising under the supervision of a physiotherapist or a fitness instructor, with the goal of promoting and continuing regular exercise in the community. ${ }^{15-18}$ CBEPs are widely used among people with chronic and episodic conditions demonstrating improvements in endurance and balance, and measures of depression for people with multiple sclerosis, cancer and stroke. ${ }^{19}{ }^{20}$ Given the increasing chronicity and multimorbidity associated with HIV, CBEPs may offer an effective and sustainable self-management strategy to improve health outcomes for PLWH. ${ }^{21}$

Researchers piloted the implementation of a 16-week fitness instructor-led CBEP and assessed the impact on health and disability before and after the programme. ${ }^{22}$ However, the experiences of participating in a CBEP from the perspective of PLWH and the extent to which a CBEP may influence ongoing long-term engagement in exercise are unknown. Our aim was to explore the experiences engaging in a CBEP from the perspective of PLWH. Specific objectives were to describe: (1) the nature and extent of exercise; (2) facilitators and barriers of engaging in a CBEP; (3) perceived benefits of participating in a CBEP on health and disability outcomes and (4) the impact of a CBEP on the long-term engagement in exercise over time.

\section{METHODS}

We conducted a descriptive qualitative study in collaboration with the Central Toronto YMCA and Toronto People with AIDS (PWA) Foundation in Toronto,
Canada. ${ }^{23}{ }^{24}$ This study was approved by the University of Toronto HIV/AIDS Research Ethics Board.

Our study builds on a pilot study that explored the implementation of a CBEP with the aim to reduce disability and improve health for PLWH. ${ }^{22}$ The CBEP included a combination of aerobic, resistance, flexibility and balance training for $90 \mathrm{~min}$, three times per week, for 16 weeks at the YMCA in Toronto, Canada. ${ }^{22}$ The intervention was specifically tailored to each participant depending on their individual goals, abilities and interests. Hence, the intensity, type and time of each form of exercise varied among participants. Exercise sessions were supervised and progressed weekly by a fitness instructor. Participants were asked to attend monthly educational self-management sessions focused on topics including exercise, healthy eating, role of occupational therapy and complementary and alternative therapies for PLWH. ${ }^{22}$ Participants received a 16-week YMCA membership for the duration of the study.

We recruited adults (18 years of age or older) living with HIV who participated in (but did not need to complete) the CBEP. ${ }^{22}$ We contacted participants from the pilot study by email or telephone who agreed to be contacted about future phases of research. Members of the research team identified themselves to potential participants as students in the Department of Physical Therapy at the University of Toronto (CAM, KJH, SRK, TBK and CFMY) who were advised by a faculty advisor throughout the research $(\mathrm{KKO})$. Face-to-face interviews were conducted at a community-based organisation (Toronto PWA Foundation), the YMCA or the University of Toronto based on the preference of participants. Five members of the team (CAM, KJH, SRK, TBK and CFMY) conducted the interviews using a semistructured interview guide (see online supplementary additional file 1$).^{25}$ Specifically, we asked participants about the strengths and limitations of the programme, if and how personal and environmental factors influenced their participation in the CBEP, perceived benefits (if any) and how the CBEP influenced their ongoing engagement in exercise after the programme. One team member interviewed and the other took field notes. Interviews were audio recorded and transcribed verbatim.

We administered a self-reported demographic questionnaire followed by the Rapid Assessment of Physical Activity (RAPA) Scale, a nine-item questionnaire that describes the frequency and intensity a participant spends in vigorous or moderate aerobic activity within a week (RAPA 1) and the frequency a participant engages in strength and flexibility activities within a week (RAPA 2). ${ }^{26}$

\section{Data analysis}

We conducted a thematic analysis examining transcripts line by line to create codes we interpreted as key concepts related to experiences with the CBEP programme. ${ }^{27} 28$ Three transcripts were independently coded by all members of the team who subsequently 
met to collectively review codes and establish a preliminary coding scheme. Pairs of researchers independently coded the remaining transcripts, met to discuss their coding interpretations and created analytic memos for each transcript. We clustered the codes into broader categories; defined each category, discussed relationships between categories and grouped categories into broader themes. ${ }^{27}$ We used NVivo V.10 qualitative software for data management (QSR International. NVivo qualitative data analysis software. V.10; 2012) ${ }^{29}$ We organised the themes and categories into a framework to describe the experiences of participating in the CBEP from the perspective of PLWH.

For the demographic questionnaire, we calculated median and IQRs for continuous variables and frequencies and per cent for categorical variables. We calculated the median (IQR) for RAPA 1 scores ranging from 1 (least active) to 7 (most active) and median (IQR) RAPA 2 scores ranging from 0 (not engaging in strength and flexibility) to 3 (doing strength and flexibility activities) ${ }^{26}$ Higher RAPA scores indicated greater frequency, intensity and duration of activity. ${ }^{26}$

\section{RESULTS}

Of the 15 individuals who were approached and met the inclusion criteria, 11 responded and agreed to participate. Eleven adults living with HIV participated in a 3090 min interview between February and June 2016. The majority were men $(64 \%)$, median age of 52 years, with a median of five self-reported concurrent health conditions in addition to HIV (table 1). Nine participants (82\%) completed the CBEP.

Interviews occurred at a median of 6 months after the CBEP. At the time of the interviews, $10(91 \%)$ participants were still exercising, $9(90 \%)$ of whom were exercising to a lesser extent than during the CBEP (table 2). At the time of the interview, four participants (36\%) reported actively engaging in aerobic and anaerobic (strength and flexibility) exercise (table 3 ).

\section{Framework of experiences of PLWH participating in a CBEP}

The framework describes participants' experiences across three time phases (before, during and after the CBEP) (figure 1). The perceived impact of the CBEP influenced the intention to, engagement in and sustainability of exercise among participants. Intrinsic and extrinsic contextual factors could facilitate or hinder participants' participation in exercise throughout all phases of the CBEP. The episodic nature of HIV and multimorbidity further influenced engagement and re-engagement in exercise throughout the continuum.

\section{Phase I: prior to the CBEP}

Intention to join

Participants expressed different motivators for joining the CBEP. Programme attributes that influenced the

\begin{tabular}{|c|c|}
\hline Characteristic & $\begin{array}{l}\text { Number of } \\
\text { participants }\left(\%^{\star}\right)\end{array}$ \\
\hline \multicolumn{2}{|l|}{ Gender } \\
\hline Man & $7(64 \%)$ \\
\hline Woman & $1(9 \%)$ \\
\hline Othert & $3(27 \%)$ \\
\hline Age (years), median (IQR) & 52 years $(48,60)$ \\
\hline Year of HIV diagnosis, median (IQR) & $2004(1990,2008)$ \\
\hline Currently taking antiretroviral therapy & $11(100 \%)$ \\
\hline Viral load undetectable & $5(45 \%)$ \\
\hline \multicolumn{2}{|l|}{ Partnership status } \\
\hline Married or common law & $4(36 \%)$ \\
\hline Widowed & $2(18 \%)$ \\
\hline Single & $4(36 \%)$ \\
\hline Separated or divorced & $1(9 \%)$ \\
\hline Lived alone & $8(73 \%)$ \\
\hline \multicolumn{2}{|l|}{ Current employment status } \\
\hline Working, volunteering or student & $7(64 \%)$ \\
\hline Retired & $1(9 \%)$ \\
\hline Not working & $3(27 \%)$ \\
\hline \multicolumn{2}{|c|}{ Household income per year (Canadian dollars) } \\
\hline$<\$ 39999$ & $5(45 \%)$ \\
\hline$\$ 40000-69999$ & $2(18 \%)$ \\
\hline$\$ 70000-999999$ & $2(18 \%)$ \\
\hline$\$ 100000-150000$ & $2(18 \%)$ \\
\hline $\begin{array}{l}\text { Median number of concurrent health } \\
\text { conditions in addition to HIV (IQR) }\end{array}$ & $5(4,6)$ \\
\hline \multirow{2}{*}{\multicolumn{2}{|c|}{$\begin{array}{l}\text { Most commonly self-reported concurrent health conditions. } \\
\text { Number of participants living with... }\end{array}$}} \\
\hline & \\
\hline Mental health condition & $6(55 \%)$ \\
\hline Bone and joint disorder & $5(45 \%)$ \\
\hline High cholesterol & $4(36 \%)$ \\
\hline Neurocognitive decline & $4(36 \%)$ \\
\hline Addiction & $3(27 \%)$ \\
\hline Human papillomavirus & $3(27 \%)$ \\
\hline Peripheral neuropathy & $3(27 \%)$ \\
\hline
\end{tabular}

intention to join the CBEP included its reputability associated with a research study and specificity of the programme for PLWH: "The real plus about [the] research ... was that it structured it for [PLWH]" (INT-10). The accessibility of the YMCA location, fitness instructors and having membership fees waived for participating in the research were additional motivators for joining the programme. Furthermore, the CBEP provided participants the chance to initiate exercise routines and healthy life choices: "[...] when I first learned of the study, I was really excited because I saw it as an opportunity to really, get serious about physical exercise and diet" (INT-9).

Goals

Prior to participating in the CBEP, participants individually set goals with their fitness instructor to define their 
desired outcomes of the programme. Examples of goals participants articulated in the interviews included: gaining strength and muscle mass, managing weight, reducing pain and improving energy. Some goals were to address disability and represented motivators to facilitate participation in the CBEP: "I wanted to build up muscle, I knew I had a knee problem, so I wanted to ease the pain in my knee" (INT-4).

Table 2 Exercise characteristics of participants related to the CBEP $(n=11)$

\begin{tabular}{|c|c|}
\hline Characteristics & $\begin{array}{l}\text { Number of } \\
\text { participants }\left(\%{ }^{\star}\right)\end{array}$ \\
\hline $\begin{array}{l}\text { Time since CBEP participation, } \\
\text { median (IQR) }\end{array}$ & 6 months $(4,8)$ \\
\hline \multicolumn{2}{|c|}{ Self-reported level of exercise prior to the CBEP } \\
\hline Not engaging in exercise & $4(36 \%)$ \\
\hline Exercising $<2$ times per week & $5(45 \%)$ \\
\hline Exercising $\geq 2$ times per week & $2(18 \%)$ \\
\hline \multicolumn{2}{|c|}{$\begin{array}{l}\text { Self-reported level of exercise immediately after completing } \\
\text { the CBEP }\end{array}$} \\
\hline $\begin{array}{l}\text { Exercised regularly } \geq 2 \text { times } \\
\text { per week }\end{array}$ & $3(27 \%)$ \\
\hline $\begin{array}{l}\text { Exercised not regularly }<2 \text { times } \\
\text { per week }\end{array}$ & $3(27 \%)$ \\
\hline Did not exercise & $5(45 \%)$ \\
\hline \multicolumn{2}{|c|}{$\begin{array}{l}\text { Self-reported level of exercise at time of interview as } \\
\text { compared to participant exercise level during the CBEP }\end{array}$} \\
\hline Exercising less & $9(82 \%)$ \\
\hline Exercising more & $1(9 \%)$ \\
\hline Exercising similarly to CBEP & $1(9 \%)$ \\
\hline
\end{tabular}

Phase II: during the CBEP

\section{Nature and extent of exercise}

Participants engaged in individualised exercise regimes ranging from $45 \mathrm{~min}$ to $2 \mathrm{~h}$ per session, two to five times per week, for 16 weeks. Exercises varied including weightlifting, endurance, flexibility and balance training. Participants met with their fitness instructors once weekly for supervision and progression of their programmes, and continuing their exercise programmes independently for the remainder of the week. One participant described his experience of engaging with the CBEP:

I met someone every week and I could go over what was working and what wasn't working and my routine or regimen was changed a few times, plus I got things to do while I was traveling. Or else I wouldn't have done anything because you're on the road [...]. (INT-11)

\section{Accountability}

Several participants described a sense of accountability towards the CBEP, specifically a responsibility to the research, their fitness instructors and to themselves. This feeling of obligation motivated participants to continue their participation with the CBEP:

I promised I would go every week, and I promised I would work two times on my own, and I managed for the most part I think to do all those things. So the fact that we made all those kinds of promises to the research, I think helped. (INT-10)

Another participant described the accountability he felt towards himself and to other PLWH:

I'm the only one alive. Everyone else died. And I think it's part of my pledge or promise to those that have

Table 3 Self-reported level of physical activity as measured by the RAPA Questionnaire

\section{RAPA Classification (RAPA Interpretation)}

Number of participants $(\% *)$

\section{Aerobic activity}

Under-active regular-light activities

(Performs some light physical activity every week)

Under-active regular activities

(Performs moderate physical activities every week but $<30$ min a day or 5 days a week OR performs vigorous physical activities every week, but $<20 \mathrm{~min}$ a day or 3 days a week)

Active

(Performs $30 \mathrm{~min}$ or more a day of moderate physical activities, 5 or more days a week OR

performs $20 \mathrm{~min}$ or more a day of vigorous physical activities, 3 or more days a week)

\section{Anaerobic activity (measured as done more than once per week)}

Strength training only

(Performs activities to increase muscle strength, such as lifting weights or callisthenics, once a week or more)

Flexibility only

(Performs activities to improve flexibility, such as stretching or yoga, once a week or more)

Strength and flexibility

Neither strength nor flexibility

${ }^{*}$ Percentages may not add up to $100 \%$ due to rounding to nearest $\%$.

RAPA: Rapid Assessment of Physical Activity. 


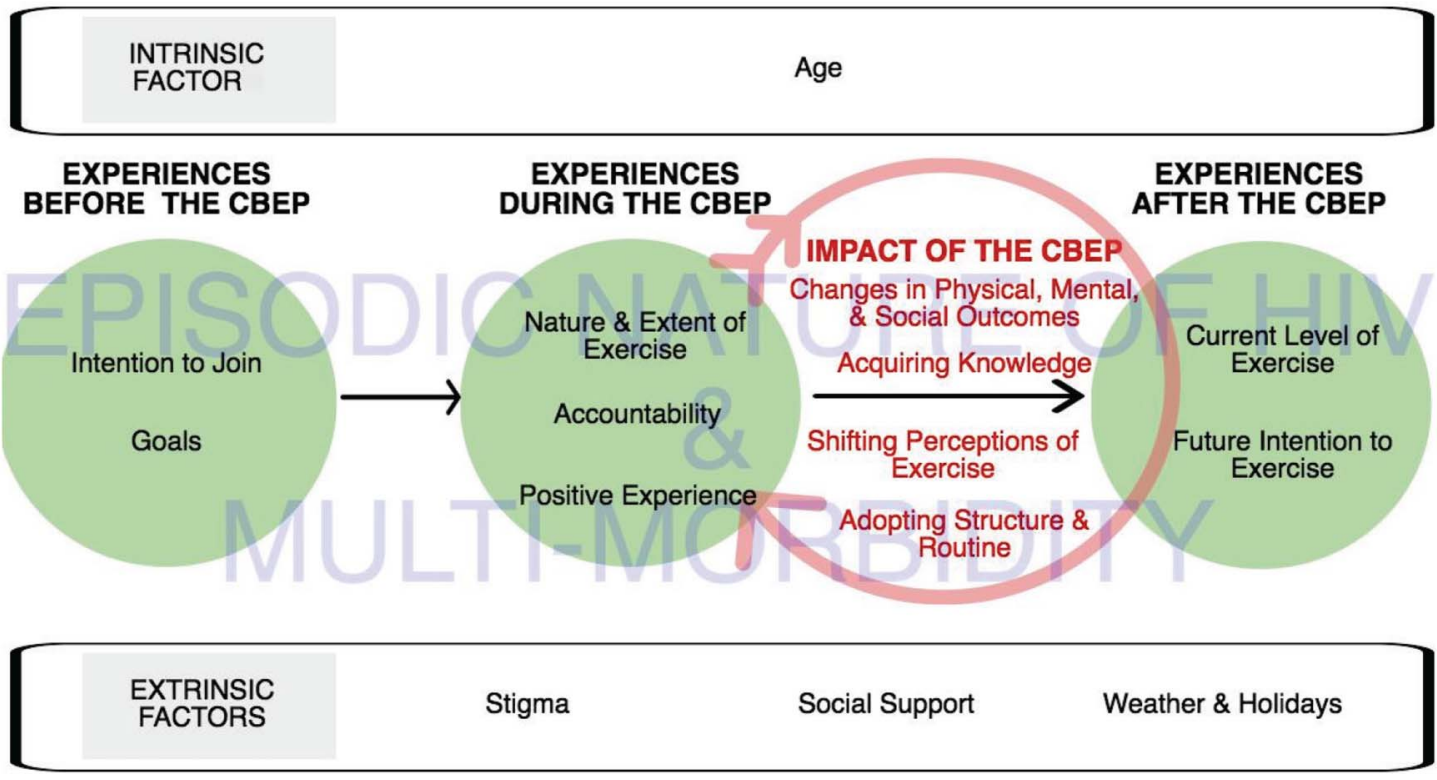

Figure 1 Framework of experiences of adults living with HIV participating in a CBEP. This framework describes participants' experiences across three time phases (before, during and after the CBEP) and the perceived impact during and after the CBEP. Intrinsic and extrinsic contextual factors facilitated or hindered participants' experience across all phases of the CBEP. The episodic nature of HIV and multimorbidity further influenced the intention to, engagement in and sustainability of exercise throughout the continuum. CBEP, community-based exercise programme.

already died from HIV is to look after myself for as long as I can, because [...] by living as good a life as I can, I'm paying homage to the fact that they weren't able to. I'm not wasting the years, or at least I'm trying not to. (INT-3)

\section{Positive experience}

Positive features of the CBEP included the environment and fitness instructors who acted as motivators to continue exercising throughout the CBEP.

\section{Environment and atmosphere}

Participants described the YMCA atmosphere as nonjudgemental, accessible and a place of familiarity:

It's also encouraging $[\ldots]$ that there are all sorts of ages, shapes, and sizes and limitations at the $\mathrm{Y}$ and there is no judgment. It's just a place where people go to try and optimize their health benefit. (INT-3)

However, several participants disliked the noise at the facility: "I loved the facility; I just don't like it when there's all the screaming kids" (INT-9).

\section{Fitness instruction}

A key component to participants' positive experiences was the fitness instructors' openness to learn about HIV, and their ability to modify activities based on individual needs. Participants described a fondness of the fitness instructors' flexibility during fluctuating periods of illness and wellness: "The fitness trainer was just wonderful, both in terms of the instruction, but also her understanding, her sensitivity [...] to my life situation" (INT-9). Fitness instructors provided ongoing support and motivation throughout the CBEP.
Impact of the CBEP

\section{Perceived changes to physical, mental and social health}

The majority of participants described improvements in their physical, mental and social health which encouraged them to remain engaged during and after the CBEP: "I did appreciate the benefits of it. That's what motivated me to continue" (INT-8). See table 4 for a detailed overview of perceived changes in health outcomes.

\section{Acquiring knowledge}

Participants' experiences were enhanced by their increased knowledge about exercise. They learnt how to safely use exercise equipment and about the physical and mental benefits of exercise from the educational sessions and fitness instructors. However, a large impact of the CBEP on knowledge acquisition appeared to emerge from their actual perceived benefits experienced with exercise:

I think you can tell people that exercise is good for you until you are blue in the face, but until you experience that exercise is good for you [...] it's just not going to be real. (INT-11)

This knowledge had a sustained impact following completion of the programme: "Well the most important thing that I learned is that any type of physical activity is useful so I [have] definitely been walking more since I participated in the program" (INT-5).

\section{Shifting perceptions of exercise}

Initially, participants felt fear and apprehension regarding exercise, but after participating in the CBEP, they 
Table 4 Participants perceived changes in physical, mental and social health with the CBEP

\begin{tabular}{|c|c|c|}
\hline Area of health & $\begin{array}{l}\text { Perceived change in health with the } \\
\text { CBEP }\end{array}$ & Examples of supporting quotations \\
\hline $\begin{array}{l}\text { Physical } \\
\text { (related to the body) }\end{array}$ & $\begin{array}{l}\text { Weight loss } \\
\text { Increased muscle mass } \\
\text { Improved endurance } \\
\text { Improved energy } \\
\text { Improved function/ability to perform } \\
\text { activities of daily living } \\
\text { Decreased pain } \\
\text { Decreased fatigue } \\
\text { Improved sleep }\end{array}$ & $\begin{array}{l}\text { "I slept better, I ate better, I felt better, had more } \\
\text { energy." (INT-1) } \\
\text { "I lost all my joint pain." (INT-3) } \\
\text { "It's like, this is, it's like a life saver! So, I feel that if I } \\
\text { hadn't... been participating in this I think I would, } \\
\text { instead of losing weight and gaining muscle and } \\
\text { losing fat, that I would probably be the same as I was } \\
\text { before or even gaining more weight." (INT-4) }\end{array}$ \\
\hline $\begin{array}{l}\text { Mental } \\
\text { (related to the mind) }\end{array}$ & $\begin{array}{l}\text { Improved confidence } \\
\text { Improved self-esteem and body } \\
\text { image } \\
\text { Decreased stress and anxiety } \\
\text { Improved mood } \\
\text { Sense of accomplishment, feeling } \\
\text { proud }\end{array}$ & $\begin{array}{l}\text { "Certainly the increased energy level. Sense of } \\
\text { well-being. Sense of accomplishment." (INT-3) } \\
\text { "I looked good in front of my father, he didn't see a } \\
\text { son that was sick and who would be dying soon. He } \\
\text { saw a healthy son." (INT-2) } \\
\text { "Probably the anxiety was related to underlying } \\
\text { depression or those feelings of fear and failure, and } \\
\text { on the positive side, I found it really helped to make } \\
\text { me feel better after the exercise." (INT 7) }\end{array}$ \\
\hline $\begin{array}{l}\text { Social } \\
\text { (related to external } \\
\text { relationships) }\end{array}$ & $\begin{array}{l}\text { Improved relationships with family } \\
\text { members, partners or friends }\end{array}$ & $\begin{array}{l}\text { "It helped with building family bonds [...] going to the } \\
\text { gym gets me physically active and gives me the } \\
\text { abilities that when I leave the gym, I can go running in } \\
\text { the park with the kids." (INT-2) } \\
\text { "I met some people there that were also exercising } \\
\text { and I think this was another motivator, that I would } \\
\text { see them at the gym. So I was more motivated } \\
\text { because I was looking [forward] to see them at the } \\
\text { class or um at the treadmills or somewhere." (INT-7) }\end{array}$ \\
\hline
\end{tabular}

reported gaining confidence and knowledge, which instilled a sense of comfort, empowerment and achievement with exercise:

I think I had a lot of fears before, not knowing what I was doing $[\ldots]$ I went in really hating exercise $[\ldots] \mathrm{I}$ don't think I missed [an exercise] session, I really enjoyed it. (INT-8)

This shifting perception of exercise persisted after the CBEP:

You know it's basically like a life saver...I do it because I feel that I have been fit in terms of how I feel but also in terms of how I manage my health over time, and you know looking after myself. So I see it as a necessity. (INT-4)

Finally, after completion of the CBEP, participants viewed exercise as a necessary self-management strategy:
If it could become part of the suite of therapies available to people living with HIV that would be great. I think it should be essential of living strategies for living with HIV. (INT-9)

\section{Adopting structure and routine}

Several participants described leading busy lives with multiple work and family responsibilities and appreciated the consistency and structure provided by the CBEP: “[...] with such a chaotic life with so many volunteer obligations, it was nice three times a week to just have a morning that was just on me" (INT-11). The structure and routine was further described as a motivator to maintain participation in the CBEP: "What helped me? Probably how I structured my day, some days I went in early in the morning to do it and sometimes it was once I finished working" (INT-10). After participants completed the CBEP, they described experiencing a lack of structure and routine which contributed to their inability to continue at the same level of activity: 
"I know myself well enough to know that if I don't participate in a structured class that probably I will fall out of the pattern" (INT-3).

\section{Phase III: after the CBEP}

\section{Current level of exercise}

Interviews in this study occurred at a median of 6 months after the CBEP. Six of the 11 participants (55\%) continued their YMCA membership after the CBEP. Types of activity participants engaged in at the time of the interview included, but were not limited to: increased amounts of walking for transportation, increased use of stairs and riding bicycles. Ten participants $(91 \%)$ reported continuing to engage in exercise, nine of whom $(90 \%)$ reported exercising to a lesser extent since the CBEP. Three participants $(27 \%)$ reported not continuing with exercise in the long term due to injury, substance abuse or no given reason.

\section{Future intention to exercise}

Participants viewed exercise as an ongoing selfmanagement strategy to maintain and promote healthy living, and all expressed a desire to incorporate exercise in their future. One participant stated: "I can see myself doing this for the rest of my life" (INT-4). Furthermore, participants described using exercise as a selfmanagement strategy ageing with HIV:

[I'm] mindful of the fact that as [...] anybody gets older, if we don't maintain a degree of physical activity...It's a slippery slope-and the less you do the less you are able to do. (INT-3)

\section{Intrinsic factor}

Age

Participants described how age influenced their experiences across the CBEP. Of the nine participants who reported their age, five were $(56 \%)$ older than 50 years from a range of 38-61 years. Participants described how their age influenced their goal setting and motivation for joining the CBEP:

[...] I think exercise for me [...] is going to be about being focused on that as I age and keeping my body less frail, more mobile, those kinds of things are important to me. (INT-10)

Within the CBEP, the majority of participants expressed that their age had little to no influence on their experiences. After the CBEP, many saw ageing as a way to motivate them to remain active and decelerate ageing:

If I'm not mindful in looking after myself, the nursing home beckons, and I really don't want that. So I have to keep as active as I can so that I can stay in my own home and look after myself. (INT-3)

\section{Extrinsic factors}

Extrinsic factors in the environment including stigma, social support and weather and holidays influenced participants' experiences with exercise.

\section{Stigma}

Prior to the CBEP, participants reported concerns regarding potential stigma related to body image in a gym environment; however, during the CBEP, participants found the YMCA gym environment less stigmatising than originally feared.

So I've learned to not be intimidated by going, because whether or not it's body image issues that one can have or the fear $[\ldots]$ the idea of gym class was something that was anathema to me and going back to that environment with a lot of jocks or people that are more well-defined or whatever you will can be a little intimidating $[\ldots]$. (INT-3)

\section{Social support}

Participants described having various levels of social support, assistance and care from others during the CBEP, but did not feel connected with other study participants, despite the group monthly education sessions. Some participants preferred to exercise alone, while others expressed a desire to expand their social network and seek support from other participants. One participant used his partner as a source of continued motivation throughout the CBEP:

That sense of connection is very important so the fact that I was able to establish that in the context of that program by working out with a buddy probably gave, I got more out of it for having done it that way. (INT-10)

Participants described various levels of support from their healthcare professionals. Some felt that their health providers encouraged and motivated their participation, while others felt no influence:

One of my HIV doctors said, when he saw my MRI, oh I guess you should see a physiotherapist, and I said oh I'm part of this research project [...] and he said oh that's better than going to see a physiotherapist, do that. (INT-4)

\section{Weather and holidays}

Participants described how weather and seasonal holidays influenced their motivation and engagement in exercise. The CBEP occurred in the spring and summer months; hence, participants perceived warm weather as a facilitator that increased their activity levels. After the CBEP, participants reported that the colder weather was a barrier to exercise. In some instances, participants perceived exercise as additional work, and viewed holidays and vacations as a time to pause from their exercise programmes: 
[...] I was pretty active up until like November, umm when it started getting really cold, then I took a break over December and Christmas and then I started again in January and then it got really cold and I just had to stop. (INT-4)

\section{Episodic nature of HIV and multimorbidity}

Some participants experienced fluctuating periods of wellness and illness which resulted in periods of inactivity due to poor health. These varying episodes affected experiences across all three phases and influenced their ability to exercise and re-engage in exercise after a period of inactivity. One participant described living with the episodic nature of HIV:

I got sicker and sicker. Same way I got better and better, I just started you lose your mobility and your ability to stretch and your motivation as time goes on. And the less you do, the less you wanted to do. (INT-2).

We defined multimorbidity as the presence of one or more health conditions, in addition to living with HIV. Mental health conditions and bone and joint disorders were the most prominent (table 1). Before the CBEP, one participant described how mental health was a motivator for exercise, helping shape her goals and experiences:

Lot of [my goals] had to do with mental and emotional health, so one of the things I talked about was my anxiety and how it played out in different areas of my body so we worked on some exercises that would just help me relax. (INT-5)

Multimorbidity was dually experienced as a barrier to exercise. Two participants (18\%) withdrew from the CBEP due to concurrent health conditions, one of whom described his experience with addiction disrupting his ability to engage in exercise:

[The CBEP] was complete stop. It was an immediate plunge back into abuse. I mean, maybe it was a relapse for, for the first few days but then it just became [...] again it became my choice. I chose [...] to go ever deeper into, my substance abuse. (INT-9)

The flexibility offered by the programme and the fitness instructor was helpful for participants to deal with and overcome the unpredictable and episodic nature of multimorbidity and HIV during the CBEP:

And one of the things I most appreciated was the flexibility, you know it wasn't, you know I was concerned that [fitness instructors] would dictate what the routine is but no, there was complete flexibility. (INT-9)

Several participants described experiencing an improvement with their multimorbidity after their engagement in the CBEP, ultimately leading to the desire to incorporate exercise as a lifestyle strategy in their future. However, as time progressed, some participants struggled with complications of HIV and multimorbidity and expressed difficulty sustaining and returning to their exercise regimens.

Finally, the majority of participants described the need for return-to-exercise strategies. One participant stated: "I guess the one goal I had was continuing to exercise after the program. And I did. But, I got sick [...], once you lose the rhythm, you really lose the rhythm" (INT-11).

\section{DISCUSSION}

To the best of our knowledge, this is the first qualitative study to explore the experiences of participating in a CBEP from the perspectives of PLWH. Perceived benefits of the CBEP were influential in promoting adherence to exercise and adoption of exercise as a long-term self-management strategy. Engagement in exercise across the phases in our framework may be considered analogous to the precontemplation, contemplation, preparation (before CBEP), action (during CBEP) and maintenance (after CBEP) stages of behaviour change in the transtheoretical model (TTM) ${ }^{30-32}$ Earlier stages of the TTM, specifically readiness to engage in exercise, have been explored from the perspectives of PLWH highlighting the contemplation phase when living with HIV and complex multimorbidity. ${ }^{33}$ Our framework depicts how PLWH transition through stages of exercise behaviour changes (contemplation and preparation) and further considers the phases during (action) and after (maintenance) of a formalised CBEP, exploring the potential to adopt exercise as a self-management strategy living with HIV. Strengthening the impact of a CBEP to yield positive experiences during a CBEP can subsequently promote maintenance of exercise for PLWH post-CBEP.

Participants described how perceived benefits experienced during the CBEP promoted adherence to, and ongoing maintenance with, exercise. Improvements in physical, mental and social health were similarly reported in systematic review evidence on the effects of exercise for PLWH. ${ }^{7} 9103435$ However, acquiring knowledge that mitigated uncertainty with exercise and incorporating exercise as a structure and routine were additional benefits articulated by participants that positively influenced their engagement with exercise. These additional benefits may be attributed to the YMCA environment, weekly supervision from a fitness instructor and the monthly education sessions. ${ }^{36}$ Similar positive features of CBEPs were documented when individuals with pulmonary disease, multiple sclerosis and stroke engaged in CBEPs. ${ }^{37-39}$ Future programmes should consider the importance of education about the safety and effectiveness of exercise, discussing pre-existing perceptions of exercise and offering support for adopting exercise as part of a regular routine. Future research may explore the extent to which these features of a CBEP 
may influence long-term sustainability of engaging in exercise and the potential benefits of a combined CBEP programme with PLWH and other chronic illnesses.

The episodic nature of HIV and multimorbidity was an underlying factor that influenced engagement and re-engagement in exercise after a period of inactivity for PLWH. Mental health and addiction issues were examples of concurrent health conditions that influenced ongoing participation in exercise. Knowledge among fitness instructors about the episodic nature of HIV, their supportive approach to adapting the CBEP to accommodate changes in health of participants along with the inclusive and accommodating nature of the YMCA, facilitated engagement in the CBEP. PLWH who are medically stable can safely exercise two to four times weekly, at $40-60 \%$ heart-rate-reserve, for 30-120 min per session, using a variety of types of exercise activity. ${ }^{40} 41$ Knowledge among health providers about the safety and effectiveness of exercise can help to ensure that they adequately promote participation in exercise in a manner that recognises, supports and accommodates the needs of PLWH while offering variable ways to maximise engagement despite fluctuations in health.

Of the 11 participants, 10 remained physically active after the programme ended, 9 of whom were less active than during the CBEP. Nevertheless, ongoing engagement in physical activity among almost all participants may be considered a success of the intervention. Some participants expressed challenges in returning to exercise after episodes of illness or multimorbidity. Fitness instructors adjusted the programmes according to the health of participants. Future programmes should consider strategies to help PLWH return to exercise or maintain exercise despite the potentially episodic nature of HIV and multimorbidity.

Our study builds on a pilot study that examined the process and implementation of a CBEP for PLWH. ${ }^{22}$ Using a qualitative approach, we were able to describe the experiences, influencing factors, and perceived impact of community-based exercise while illustrating the complex interactions between the components and underlying influence of the episodic nature of HIV and multimorbidity. This framework can be used by clinicians, representatives from the fitness community and PLWH to inform the understanding of experiences of engaging in exercise, and the features to consider when designing and implementing future exercise programmes for PLWH.

Our study possesses some limitations. The majority of participants completed the CBEP and thus may have represented a 'healthy' community-dwelling sample of PLWH. Nevertheless, many described having to cancel appointments or scale back on exercise due to periods of illness. Participants in this study were primarily men living in downtown Toronto who completed the CBEP and agreed to participate in an interview. Hence, the transferability of these findings to women, PLWH living in rural settings and for those who withdrew or were lost to follow-up are less clear. Finally, our aim was not to achieve saturation, but rather to obtain a rich description of experiences in the CBEP. Nevertheless, we ceased the interviews at 11 , which we observed as the point when no new categories emerged.

\section{CONCLUSIONS}

Results describe experiences before, during and after engaging in a CBEP from the perspective of adults living with HIV. The positive impacts of the CBEP and the episodic nature of HIV and multimorbidity influenced engagement in and sustainability of exercise among PLWH. Future CBEPs should include strategies to accommodate potential fluctuations in health and promote return-to-exercise strategies to assist with re-engagement in exercise after an episode of illness. This framework may be used by healthcare professionals when recommending and discussing exercise with PLWH and can inform the design of future CBEPs in order to promote imitation and sustained engagement in physical activity among PLWH.

\section{Author affiliations}

${ }^{1}$ Department of Physical Therapy, University of Toronto, Toronto, Ontario, Canada

${ }^{2}$ Rehabilitation Sciences Institute (RSI), University of Toronto, Toronto, Ontario, Canada

${ }^{3}$ Institute of Health Policy, Management and Evaluation (IHPME), University of Toronto, Toronto, Ontario, Canada

Acknowledgements The authors acknowledge the Central Toronto YMCA staff for their collaboration in this work in the Community-Based Exercise Programme Study, including Mehdi Zobeiry, Katie Lowe, Maria Rapalini, Ivan Ilic, Emily Sas, Liam Dick, Dexter Wilson, Helen Trent, Katharine Stanbridge, Christine Hsu, Cristina Granados and Letizia Lepore. The authors acknowledge the Toronto People with AIDS (PWA) Foundation (Chris Godi) for their support with recruitment. They acknowledge Patrick Jachyra, Teaching Assistant, from the University of Toronto, who assisted with development of the protocol.

Contributors KKO $(\mathrm{PhD})$ designed the study and provided guidance throughout the research process and possesses expertise in qualitative methodology and HIV and exercise research. KKO supervised CAM, KJH, SRK, TBK and CFMY who developed the protocol, collected and analysed the data and drafted the manuscript in partial fulfilment of requirements for an MScPT degree at the University of Toronto. CAM, KJH, SRK, TBK and CFMY (MScPT students) developed skills in qualitative research methodology including attending lectures; completing readings on qualitative research study design; understanding steps of recruitment, data collection and analysis; completing a literature review; developing the research protocol; interview guide and demographic questionnaire and considering the ethical issues associated with this research. All steps were closely reviewed and guided by KKO (advisor). All authors read and approved the final manuscript.

Funding This research was funded by a Connaught New Researcher Award at the University of Toronto. KKO is supported by a New Investigator Award from the Canadian Institutes of Health Research (CIHR).

Competing interests None declared.

Patient consent Obtained.

Ethics approval University of Toronto HIV/AIDS Research Ethics Board.

Provenance and peer review Not commissioned; externally peer reviewed.

Data sharing statement Data collected and analysed during the study are not publicly available in accordance with our study protocol that was approved by 
the University of Toronto HIV/AIDS Research Ethics Board. The data may be available on reasonable request by contacting the corresponding author

Open Access This is an Open Access article distributed in accordance with the Creative Commons Attribution Non Commercial (CC BY-NC 4.0) license, which permits others to distribute, remix, adapt, build upon this work noncommercially, and license their derivative works on different terms, provided the original work is properly cited and the use is non-commercial. See: http:// creativecommons.org/licenses/by-nc/4.0/

\section{REFERENCES}

1. Antiretroviral Therapy Cohort Collaboration. Causes of death in HIV-1-infected patients treated with antiretroviral therapy, 19962006: collaborative analysis of 13 HIV Cohort studies. Clin Infect Dis 2010;50:1387-96.

2. Rodriguez-Penney AT, ludicello JE, Riggs PK, et al. Co-morbidities in persons infected with HIV: increased burden with older age and negative effects on health-related quality of life. AIDS Patient Care STDS 2013;27:5-16.

3. Oursler KK, Goulet JL, Crystal S, et al. Association of age and comorbidity with physical function in HIV-infected and uninfected patients: results from the Veterans Aging Cohort Study. AIDS Patient Care STDS 2011;25:13-20.

4. O'Brien KK, Bayoumi AM, Strike C, et al. Exploring disability from the perspective of adults living with HIV/AIDS: development of a conceptual framework. Health Qual Life Outcomes 2008;6:76.

5. O'Brien KK, Davis AM, Strike C, et al. Putting episodic disability into context: a qualitative study exploring factors that influence disability experienced by adults living with HIV/AIDS. J Int AIDS Soc 2009; $12: 5$.

6. Worthington C, Myers T, O'Brien K, et al. Rehabilitation in HIV/AIDS development of an expanded conceptual framework. AIDS Patient Care STDS 2005;19:258-71.

7. Gomes Neto M, Ogalha C, Andrade AM, et al. A systematic review of effects of concurrent strength and endurance training on the health-related quality of life and cardiopulmonary status in patients with HIV/AIDS. Biomed Res Int 2013;2013:319524.

8. Clingerman E. Physical activity, social support, and health-related quality of life among persons with HIV disease. J Community Health Nurs 2004;21:179-97.

9. O'Brien K, Tynan AM, Nixon S, et al. Effects of progressive resistive exercise in adults living with HIV/AIDS: systematic review and meta-analysis of randomized trials. AIDS Care 2008;20:631-53.

10. O'Brien K, Tynan AM, Nixon S, et al. Effectiveness of aerobic exercise for adults living with HIV: systematic review and meta-analysis using the Cochrane Collaboration protocol. BMC Infect Dis 2016;16:182. http://www.biomedcentral.com/1471-2334/ $16 / 182$.

11. Schuelter-Trevisol F, Wolff FH, Alencastro PR, et al. Physical activity: do patients infected with HIV practice? How much? A systematic review. Curr HIV Res 2012;10:487-97.

12. Rimmer JH, Wang E, Smith D. Barriers associated with exercise and community access for individuals with stroke. J Rehabil Res Dev 2008;45:315-22.

13. Hawley-Hague $\mathrm{H}$, Horne $\mathrm{M}$, Campbell $\mathrm{M}$, et al. Multiple levels of influence on older adults' attendance and adherence to community exercise classes. Gerontologist 2014;54:599-610.

14. Grant B. 'Against the grain': over 75 years and joining a community exercise programme. Qual Res Sport Exerc Health 2012;4:1-14.

15. Salbach NM, Howe JA, Brunton K, et al. Partnering to increase access to community exercise programs for people with stroke, acquired brain injury, and multiple sclerosis. J Phys Act Health 2014:11:838-45.

16. Smith $\mathrm{C}$, Olson $\mathrm{K}$, Hale LA, et al. How does fatigue influence community-based exercise participation in people with multiple sclerosis? Disabil Rehabil 2011;33:2362-71.

17. Stuart M, Benvenuti F, Macko R, et al. Community-based adaptive physical activity program for chronic stroke: feasibility, safety, and efficacy of the Empoli Model. Neurorehabil Neural Repair 2009;23:726-34.

18. Winward C. Supporting community-based exercise in long-term neurological conditions: experience from the Long-term Individual Fitness Enablement (LIFE) project. Clin Rehabil 2011;25:579-87.
19. Moore SA, Hallsworth K, Jakovljevic DG, et al. Effects of community exercise therapy on metabolic, brain, physical, and cognitive function following stroke: a randomized controlled Pilot trial. Neurorehabil Neural Repair 2015;29:623-35.

20. Ploughman M, Shears J, Harris C, et al. Effectiveness of a novel community exercise transition program for people with moderate to severe neurological disabilities. Neurorehabilitation 2014;35:105-12.

21. O'Brien KK, Solomon P, Trentham B, et al. Evidence-informed recommendations for rehabilitation with older adults living with HIV: a knowledge synthesis. BMJ Open 2014;4:e004692.

22. O'Brien K, Nayar A, Bayoumi A, et al. eds. Implementing a community-based exercise intervention to improve the health of adults living with HIV: a pilot study. Annual Canadian Conference on HIVIAIDS (CAHR Conference), Winnipeg; May 12-15, 2016; 2016.

23. Toronto People with AIDS Foundation. Toronto people with AIDS Foundation. 2015; http://www.pwatoronto.org/ (accessed 5 Oct 2015)

24. YMCA of Greater Toronto. YMCA of Greater Toronto. 2015; http:// www.ymcagta.org/en/index.html (accessed 5 Oct 2015).

25. Gill $\mathrm{P}$, Stewart K, Treasure E, et al. Methods of data collection in qualitative research: interviews and focus groups. Br Dent $J$ 2008;204:291-5.

26. Topolski TD, LoGerfo J, Patrick DL, et al. The Rapid Assessment of Physical Activity (RAPA) among older adults. Prev Chronic Dis 2006;3:A118.

27. Carpenter C, Suto M. Qualitative research for occupation and physical therapists: a practical guide. 1st edn. Oxford: Wiley-Blackwell, 2008

28. Braun V, Clarke V. Using thematic analysis in psychology. Qual Res Psychol 2006;3:77-101.

29. QSR International. NVivo Qualitative Data Analysis Software. Version 10; 2012. () QSR International Pty Ltd. http://www. qsrinternational.com/

30. Basta TB, Reece M, Wilson MG. Predictors of exercise stage of change among individuals living with HIV/AIDS. Med Sci Sports Exerc 2008;40:1700-6

31. Basta TB, Reece M, Wilson MG. The transtheoretical model and exercise among individuals living with HIV. Am J Health Behav 2008;32:356-67.

32. Prochasaa JO, Redding CA, Evers KE. The transtheoretical Model and Stages of Change. In: Glanz K, Rimer BK, Lewis FM, eds. Health behaviour and health education. 3rd edn. San Francisco: Jossey-Vass, 2002: 99-120.

33. Simonik A, Vader K, Ellis D, et al. Are you ready? Exploring readiness to engage in exercise among people living with HIV and multimorbidity in Toronto, Canada: a qualitative study. BMJ Open 2016;6:e010029. http://bmjopen.bmj.com/content/6/3/e010029.full.

34. Gomes-Neto M, Conceicao CS, Oliveira Carvalho V, et al. A systematic review of the effects of different types of therapeutic exercise on physiologic and functional measurements in patients with HIV/AIDS. Clinics (Sao Paulo) 2013;68:1157-67.

35. Gomes Neto M, Conceicao CS, Oliveira Carvalho V, et al. Effects of combined aerobic and resistance exercise on exercise capacity, muscle strength and quality of life in HIV-infected patients: a systematic review and meta-analysis. PLOS One 2015;10:e0138066.

36. Harris M. Exercise in HIV infection: the value, the difficulties in community-based implementation. Arch Phys Med Rehabil 2011:92:1734.

37. Learmonth YC, Marshall-Mckenna R, Paul L, et al. A qualitative exploration of the impact of a 12-week group exercise class for those moderately affected with multiple sclerosis. Disabil Rehabil 2013;35:81-8

38. Reed M, Harrington R, Duggan A, et al. Meeting stroke survivors perceived needs: a qualitative study of a community-based exercise and education scheme. Clin Rehabil 2010;24:16-25.

39. de Sousa Pinto JM, Martin-Nogueras AM, Morano MT, et al. Chronic obstructive pulmonary disease patients' experience with pulmonary rehabilitation: a systematic review of qualitative research. Chron Respir Dis 2013;10:141-57.

40. Grace J, Semple S, Combrink S. Exercise therapy for human immunodeficiency virus/AIDS patients: guidelines for clinical exercise therapists. J Exerc Sci Fit 2015;13:49-56.

41. Yahiaoui A, McGough EL, Voss JG. Development of evidence-based exercise recommendations for olde HIV-infected patients. J Assoc Nurses AIDS Care 2012;23:204-19. 